\title{
Comparison of Prediction Between TIMI (Thrombolysis in Myocardial Infarction) Risk Score and Modified TIMI Risk Score in Discharged Patients From Emergency Department With Atypical Chest Pain
}

\author{
Mohsen Abbasnezhad ${ }^{1}$; Hassan Soleimanpour ${ }^{2,}$; Mohamadreza Sasaie ${ }^{3}$; Samad EJ Golzari ${ }^{4}$; \\ Saeid Safari ${ }^{5}$; Maryam Soleimanpour ${ }^{6}$; Robab Mehdizadeh Esfanjani ${ }^{7}$ \\ ${ }_{1}^{1}$ Department of Cardiology, Tabriz University of Medical Sciences, Tabriz, IR Iran \\ ${ }^{2}$ Cardiovascular Research Center, Tabriz University of Medical Sciences, Tabriz, IR Iran \\ ${ }^{3}$ Students' Research Committee, Tabriz University of Medical Sciences, Tabriz, IR Iran \\ ${ }_{5}^{4}$ Medical Philosophy and History Research Center, Tabriz University of Medical Sciences, Tabriz, IR Iran \\ 5 Department of Anesthesiology and Critical Care, Iran University of Medical Sciences, Tehran, IR Iran \\ ${ }_{7}^{6}$ Gastroenterology Research Center, Tabriz University of Medical Sciences, Tabriz, IR Iran \\ 7 Gastroenterology Research Center, Tabriz University of Medical Sciences, Tabriz, IR Iran \\ ${ }^{*}$ Corresponding Author: Hassan Soleimanpour, Cardiovascular Research Center, Tabriz University of Medical Sciences, Tabriz, IR Iran. Tel: +98-9141164134, Fax: +98-4113352078, E-mail: \\ soleimanpourh@tbzmed.ac.ir
}

Received: July 31, 2013; Revised: November 29, 2013; Accepted: January 1, 2014

Background: Chest pain is one of the most common causes of the admission to the emergency departments. It, however, can be due to numerous diseases some of which are life threatening.

Objectives: In the current study, we evaluated the prognostic value of TIMI(Thrombolysis in Myocardial Infarction) and Modified TIMI risk scores to stratify the risk for patients with atypical chest pain being discharged from the emergency department.

Patients and Methods: In a prospective-analytic study, we collected data from 1020 patients with atypical chest pain enrolled to the study. All eligible patients were visited by the emergency medicine residents who were trained for this study. Based on the criteria in both systems, the emergency medicine attending decided on either discharging or hospitalizing patients. Patients were allocated into 2 equal groups randomly. In order to predict the opposing accidents in 30 days (coronary revascularization, myocardial infarction, and all-cause death) TIMI risk scores and Modified TIMI risk scores were assessed based on TIMI risk score (0 or 1$)$ and Modified TIMI risk score (0 or 1$)$. Results: No significant difference could be observed between both groups regarding demographic characteristics, ejection fraction, left ventricle hypertrophy, TRS criteria, risk factors and the history of coronary artery stenosis. None of the atypical chest pain patients discharged based on TIMI and modified TIMI risk scores experienced any adverse events.

Conclusions: The results obtained from this study support the idea that the TIMI and modified TIMI risk scores might be valuable tools that could be used to stratify the risk of patients with atypical chest pain in the emergency department.

Keywords: Thrombolysis; Myocardial Infarction; Emergency Department; Atypical Chest Pain

\section{Background}

Chest pain is one of the most common reasons of the patients' referral to the emergency departments (EDs). It, however, can be due to numerous diseases some of which are life threatening. Cardiovascular diseases, aortic dissection, pulmonary emboli, pneumothorax, and pericarditis are some of the fatal diagnoses associated with chest pain. Acute coronary syndrome (ACS), as one of the most important causes of chest pain with a high associated mortality rate, is of great importance and should be diagnosed as early as possible (1). Approach to the chest pain, either typical or atypical, consists of a primary rapid evaluation of differentiating its being typical or a typical. Atypical chest pain cannot rule out MI especially in the female or diabetic patients; therefore, numerous studies have been performed on the more appropriate diagnosis of ACS emergency setting (2).

In other words, atypical chest pain is a common clinical problem which the physicians working in the internal medicine, emergency medicine, and cardiology departments are faced with. Atypical chest pain is mostly seen in the young female patients which is a rarely of the coronary arteries origin. However, whenever of a cardiac origin, it is associated with an additional $7 \%$ mortality rate compared with male patients at the same age. Most of these patients undergo angiography to rule out the chance of the coronary artery disease (3). Therefore, one of the major problems is to determine if the existing chest pain is related to ACS or not. A definite criterion is essential to assist us in deciding on if the patient requires hospitalization for further evaluations (4-6).

Implication for health policy/practice/research/medical education:

Atypical chest pain is a common clinical problem which the physicians working in the internal, emergency, and cardiac medicine departments are faced with. In the current study we investigated the prognostic value of TIMI and Modified TIMI risk scores to risk stratify patients with atypical chest pain being discharged from the emergency department. We concluded that Both TRS and MTRS could be used for risk prediction in patients discharged with atypical chest pain from the EDs.

Copyright (C) 2014, Iranian Red Crescent Medical Journal; Published by Kowsar Corp. This is an open-access article distributed under the terms of the Creative Commons Attribution License, which permits unrestricted use, distribution, and reproduction in any medium, provided the original work is properly cited. 
TIMI Risk Score (TRS) which is used to determine the risk for patients with ACS and is mostly used for patients having chest pain syndrome with unstable angina or myocardial infarction without ST segment elevation (NSTEMI). The scoring uses seven major criteria, each measured as one score, as following (7-11):

1. Age older than 65 years

2. Coronary artery stenosis of more than $50 \%$

3. More than two cardiac disease risk factors

4. History of taking aspirin in the previous seven days

5. Incidence of more than one spells of chest pain within the previous 24 hours

6. ECG changes rather than STEMI

7. Elevated serum levels of cardiac biomarkers

Score Interpretation for TIMI scoring system would be as featured as \% risk at 14 days of all-cause mortality, new or recurrent MI, or severe recurrent ischemia requiring urgent revascularization:

Score of $0-1=4.7 \%$ risk

Score of $2=8.3 \%$ risk

Score of $3=13.2 \%$ risk

Score of $4=19.9 \%$ risk

Score of $5=26.2 \%$ risk

Score of 6-7 = at least $40.9 \%$ risk

The criteria used in Modified TIMI Risk Score (MTRS) are comparable to that of TRS; while in Modified TIMI, the scoring stays between 0-10 and a score of 5, if positive (with a total maximum score of five), reflects cardiac biomarker changes or ECG (6).

\section{Objectives}

In the current study, we evaluated the risk of the patients with atypical chest pain using previously-introduced risk scores of TIMI and Modified TIMI.

\section{Patients and Methods}

In a prospective-analytic study carried out in the ED of Shahid Madani hospital, Tabriz, IR Iran from 2011-2012, patients over 25 years old and with atypical chest pain were included. The Exclusion criteria were as following: age younger than 25 years old, diagnosed STEMI, pregnancy, hospitalization due to other reasons (chronic renal failure requiring dialysis, myocardial contusion, pregnancy), and cases for which follow-up was not practical. This study was approved by the Ethics Committee of "Tabriz University of Medical Sciences" and registered under the Code Number of 8966. After obtaining written informed consents from all patients, ECGs were taken and cardiac Troponin-I enzyme (cTnI) were checked on admission and 12 hours post-symptoms onset. To evaluate cTnI, cTnI ELISA kit (DiaPlis, USA), and to evaluate EKGs ECG monitors (Heart Screen 60-IKO, Innomed, Hungary) were used. All equipment was calibrated prior to administration. Later, patients were allocated into two equal subgroups of I and II randomly by block randomization. Patients in Groups I and II were assessed by TRS and modified TRS, respectively and those with low risk(0-1) were discharged.

All eligible patients were visited by the emergency medicine residents who were trained for this study. Hospitalization or discharge of patients were decided on by the emergency medicine attending (a same attending) based on the two systems. Follow-up via telephone for any probable unwanted complications such as MI, revascularization or death consisted of a one-month period. The data were analyzed using descriptive and deductive-statistical approaches by SPSS ver. 15 software. Variation between qualitative variables and the relation between qualitative and quantitative variables were study using Chi-square and T-test approaches, respectively. The normality of variables was checked by Kolmogorov - Smirnov Z test. Man-Whitney test was used whenever required and $\mathrm{P} \leq 0.05$ was considered statistically significant. A flow diagram of our study is showed in (Figure 1).

\section{Results}

Within the one-year period of the study,1020 patients with atypical chest pain were allocated to the study from which 20 people were excluded due to unavailability of follow-up for adverse events (MI, revascularization, death) and finally 1000 people (500 people for each group) including 595 (59.5\%) males and 405 (40.5\%) females were studied. The mean age of the studied patients was $47.72 \pm 13.59$ years (the youngest and oldest patients were 25 and 91 years old, respectively). Demographic characteristics, ejection fraction, and left ventricle hypertrophy were of no significant difference in both groups (Table 1, Figure 2).

None of the atypical chest pain patients discharged based on TIMI and modified TIMI risk scores experienced any adverse events. No statistically significant difference was observed between both groups regarding TRS criteria, risk factors and the history of coronary artery stenosis; however, a significant difference was detected regarding the number of patients using aspirin (Tables 2-4).

\section{Discussion}

From all patients with atypical chest pain referring to the $\mathrm{ED}$, almost 7\% have been reported to have ECGs with the signs of ischemia or MI and only do $6-10 \%$ of the patients have primary positive cardiac enzymes. Rest of the patients, despite not fulfilling the required diagnostic criteria, could also have ACS demanding further tests for confirming or ruling out ACS (5). TIMI Risk Score has been reported to be of high success rate in many studies. In a study carried out by Chase et al. it was suggested that the higher the TRS, the more increased the probability of one-month adverse events (death, acute myocardial infarction, and coronary revascularization) (7). In a recent study carried out by Kelly, patients having chest pain with normal ECG, TRS of zero and normal cardiac enzyme levels have been reported to be highly improbable to experience adverse events (8). 


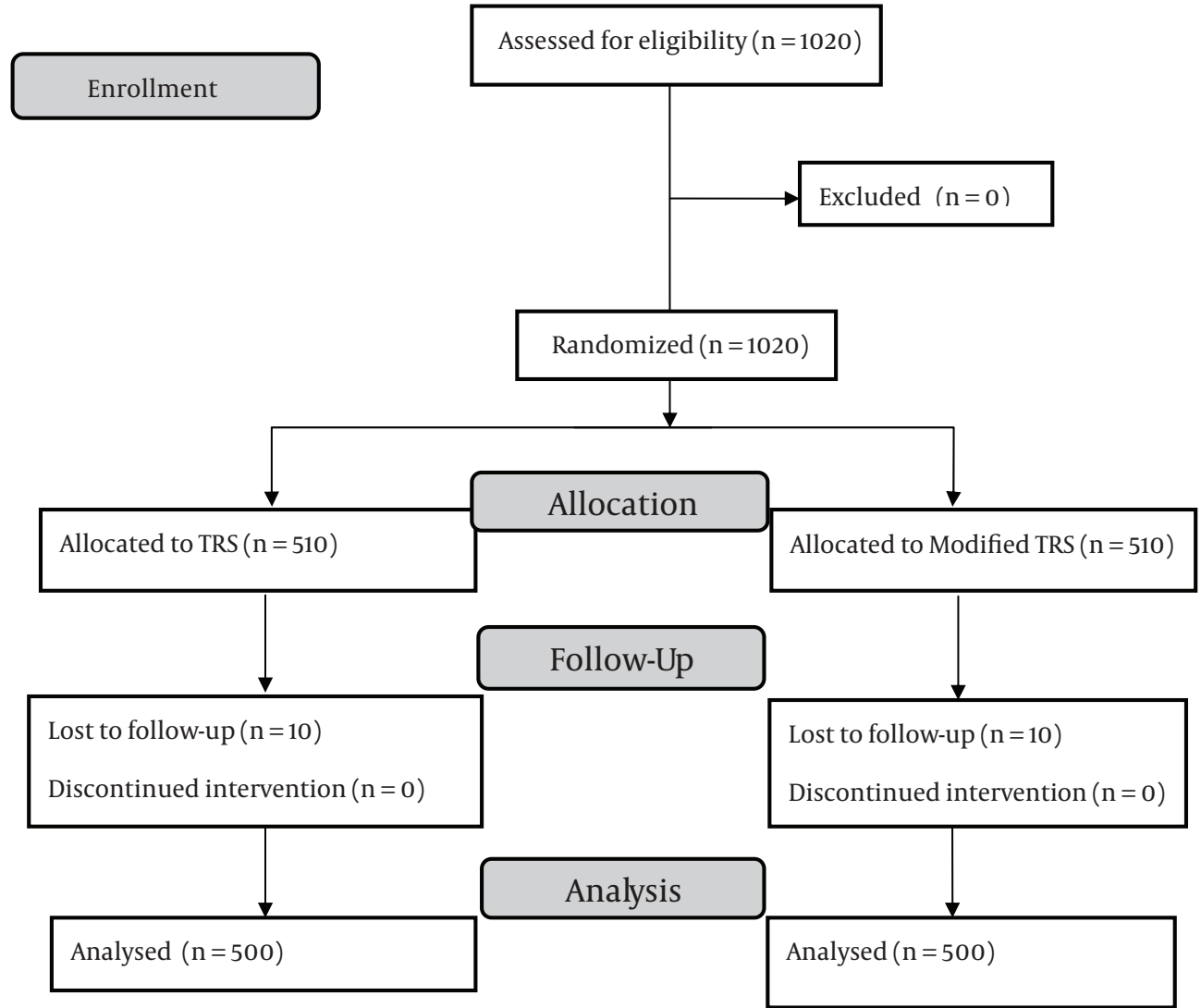

Figure 1. Flow Diagram of Study

Table 1. Demographic Characteristics and Echocardiographic Findings in Two Groups

\begin{tabular}{llll}
\hline & Group I & Group II & P value \\
\hline Age Mean \pm SD, $y$ & $47.58 \pm 56.13$ & $47.84 \pm 13.66$ & 0.761 \\
$\begin{array}{l}\text { Ejection Fraction, } \\
\text { Mean } \pm \text { SD }\end{array}$ & $57.24 \pm 4.72$ & $57.25 \pm 5.24$ & 0.983 \\
\hline
\end{tabular}

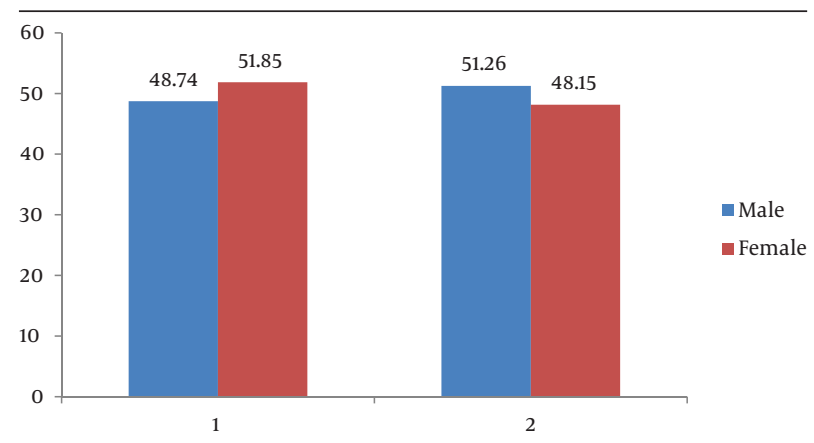

Figure 2. Female to Male Ratio in Both Groups; no Significant Difference Was Observed $(\mathrm{P}=0.766)$

Table 2. Comparison of the TIMI in Both Groups

\begin{tabular}{llll}
\hline TIMI Criteria & Group I, No. (\%) & Group II, No. (\%) & P value \\
\hline More than 3 risk factors & $16(3.2)$ & $13(2.6)$ & 0.572 \\
\hline Age over 65 years old & $51(10)$ & $39(7.8)$ & 0.185 \\
\hline Stenosis history & $9(1.8)$ & $15(3)$ & 0.215 \\
\hline ST segment changes & $8(1.6)$ & 0 & NA \\
\hline More than two chest pain reports within previous 24 hours & $11(2.2)$ & $17(3.4)$ & 0.250 \\
\hline Aspirin Consumption & $27(5.4)$ & $64(12.8)$ & $<0.0001$ \\
\hline Increased cardiac enzymes & 0 & 0 & NA \\
\hline
\end{tabular}


Abbasnezhad M et al.

\begin{tabular}{llll}
\hline Table 3. Comparison of the Risk Factors Between Two Groups & & \\
\hline Risk Factors & Group I, No. (\%) & Group II, No. (\%) & P value \\
\hline Hypertension & $165(33)$ & $148(29.6)$ & 0.246 \\
Diabet mellitus & $41(8.2)$ & $34(6.8)$ & 0.401 \\
Cigarette smokers & $97(19.4)$ & $102(20.4)$ & 0.692 \\
Familial history & $27(5.4)$ & $21(4.2)$ & 0.375 \\
Hyperlipidemia & $79(15.8)$ & $74(14.8)$ & 0.661 \\
\hline
\end{tabular}

Table 4. History of Coronary Artery Stenosis

\begin{tabular}{llll}
\hline History of Coronary artery stenosis & Group I, No. (\%) & Group II, No. (\%) & P value \\
\hline Cardiac catheterization & $3(6)$ & $3(6)$ & NA \\
Previous angioplasty & $10(2.0)$ & $4(8)$ & 0.106 \\
Coronary artery bypass graft & $2(4)$ & $10(2)$ & 0.020 \\
\hline
\end{tabular}

Although TRS enables us to classify the unselected ED chest pain population and achieve solid decisions, it is prone to missing $2-5 \%$ of the patients with myocardial infarction (4). In another study by Almagro et al. TRS was shown to be an appropriate predicted tool either in longterm or short-term in patients with atypical chest pain referring to the EDs (12). On the other hand, MTRS has been introduced as a superior scoring system of the risk evaluation in patients with undifferentiated chest pain referring to the EDs $(6,13)$. Unlike other studies, our study revealed that both TRS and MTRS have the equal predictive values in patients with atypical chest pain referring to the ED and none is superior to the other. Adverse complications could occur both in TRS and MTRS systems requiring precise clinical judgment and multi-element follow up for all patients.

\subsection{Limitations}

One of the limitations of the current study was its being single center studying only Iranian patients. In addition, we were not able to follow almost 20 patients. Another factor which may have posed selection bias leading to misclassification is the fact that our trained researchers were present in the emergency department for only 12 hours a day and seven days a week.

In conclusion Both TRS and MTRS could be used for risk prediction in patients discharged with atypical chest pain from the EDs. However, further future multi-center studies with higher sample volumes are recommended to approve the result obtained from the current study.

\section{Acknowledgements}

The authors are grateful to all the health personnel and patients who participated in the study, in addition to the data collectors, supervisors and administrative staff of Emergency department of Madani Hospital, Tabriz, Iran. Special thanks to Research Vice Chancellor Tabriz University of Medical Sciences for all the material and financial support in our study. "This article was written based on a dataset of specialty degree, registered in Tabriz University of Medical Sciences."

\section{Authors' contributions}

Mohsen Abbasnezhad, Hassan Soleimanpour, Mohamadreza Sasaie and Samad EJ Golzari, performed the clinical data collection, literature review, and drafting the manuscript. Saeid Safari, Maryam Soleimanpour and Robab Mehdizadeh Esfanjani undertook the major parts of the study design and performed the statistical analysis. All of the authors were involved in either managing the patients or writing the manuscript. All authors read and approved the final manuscript.

\section{Financial Disclosure}

There is no financial disclosure.

\section{Funding Support}

This article was supported by Research Vice Chancellor of Tabriz University of Medical Sciences, Tabriz, IR Iran.

\section{References}

1. Lyon R, Morris AC, Caesar D, Gray S, Gray A. Chest pain presenting to the Emergency Department--to stratify risk with GRACE or TIMI? Resuscitation. 2007;74(1):90-3.

2. Paul. M. , Paulman AA, Paulman JDH. 2ed ed: Lippincott Williams \& Wilkins; 2007.

3. Rasmussen JW, Grothusen JR, Rosso AL, Schwartzman RJ. Atypical chest pain: evidence of intercostobrachial nerve sensitization in Complex Regional Pain Syndrome. Pain Physician 2009;12(5):E329-34.

4. Pollack CJ, Sites FD, Shofer FS, Sease KL, Hollander JE. Application of the TIMI risk score for unstable angina and non-ST elevation acute coronary syndrome to an unselected emergency department chest pain population. Acad Emerg Med. 2006;13(1):13-8.

5. Hess EP, Perry JJ, Calder LA, Thiruganasambandamoorthy V, Body $\mathrm{R}$, Jaffe A, et al. Prospective validation of a modified thrombolysis in myocardial infarction risk score in emergency department patients with chest pain and possible acute coronary syndrome. Acad Emerg Med. 2010;17(4):368-75. 
6. Jaffery Z, Hudson MP, Jacobsen G, Nowak R, McCord J. Modified thrombolysis in myocardial infarction (TIMI) risk score to risk stratify patients in the emergency department with possible acute coronary syndrome. J Thromb Thrombolysis. 2007;24(2):137-44.

7. Chase M, Robey JL, Zogby KE, Sease KL, Shofer FS, Hollander JE. Prospective validation of the Thrombolysis in Myocardial Infarction Risk Score in the emergency department chest pain population. Ann Emerg Med. 2006;48(3):252-9.

8. Kelly AM. What is the incidence of major adverse cardiac events in emergency department chest pain patients with a normal ECG, thrombolysis in myocardial infarction score of zero and initial troponin $<=99$ th centile: an observational study? Emerg Med J.2013;30(1):15-8.

9. Conway Morris A, Caesar D, Gray S, Gray A. TIMI risk score accurately risk stratifies patients with undifferentiated chest pain presenting to an emergency department. Heart. 2006;92(9):1333-4.
10. Golabchi A, Sadeghi M, Sanei H, Akhbari MR, Seiedhosseini SM, Khosravi P, et al. Can timi risk score predict angiographic involvement in patients with st-elevation myocardial infarction? ARYA Atheroscler. 2010;6(2):69-73.

11. Lee B, Chang AM, Matsuura AC, Marcoon S, Hollander JE. Comparison of cardiac risk scores in ED patients with potential acute coronary syndrome. Crit Pathw Cardiol. 2011;10(2):64-8.

12. Garcia-Almagro FJ, Gimeno JR, Villegas M, Hurtado J, Teruel F, Cerdan MC, et al. Prognostic value of the Thrombolysis in Myocardial Infarction risk score in a unselected population with chest pain. Construction of a new predictive model. Am J Emerg Med. 2008;26(4):439-45.

13. Body R, Carley S, McDowell G, Ferguson J, Mackway-Jones K. Can a modified thrombolysis in myocardial infarction risk score outperform the original for risk stratifying emergency department patients with chest pain? Emerg Med J. 2009;26(2):95-9. 